\title{
Potential effect of smoking on semen quality through DNA damage and the downregulation of Chk1 in sperm
}

\author{
XIANGRONG CUI ${ }^{1}$, XUAN JING $^{2}$, XUEQING WU $^{1}$, ZHENQIANG WANG $^{1}$ and QIANG LI $^{1}$ \\ ${ }^{1}$ Reproductive Medicine Center, Children's Hospital of Shanxi and Women Health Center of Shanxi; \\ ${ }^{2}$ Clinical Laboratory, Shanxi Province People's Hospital, Taiyuan, Shanxi 030000, P.R. China
}

Received June 7, 2015; Accepted April 21, 2016

DOI: $10.3892 / \mathrm{mmr} .2016 .5318$

\begin{abstract}
Previous studies have found that smoking is associated with decreased male fertility via altering the quality of semen. However, the mechanism by which cigarette smoking affects semen quality remains to be fully elucidated. Heavy smoking-induced DNA damage has been reported to correlate with abnormal spermatozoa and male infertility. It has been reported that, in response to DNA damage, activation of the checkpoint kinase 1 (Chk1) facilitates S and G2 checkpoint arrest. The aim of the present study was to investigate the expression levels of Chk1 in sperm cells of smoking and non-smoking men, and to further examine the correlation between DNA fragmentation rates and the expression levels of Chk1 with smoking. The present study was performed on a cohort of 841 smoking men and 287 non-smoking men. In the investigation, sperm concentration, motility, viability, seminal plasma zinc concentration, acrosin activity and sperm DNA fragmentation were examined. The gene and protein expression levels of Chk1 were detected using reverse transcription-quantitative polymerase chain reaction and western blot analyses, respectively. It was observed that the progressive motility of the sperm was significantly decreased in the moderate and heavy smoking groups, whereas no significant changes were observed in the mild smoking group. The sperm in the medium-term smoking group had significantly decreased progressive motility, and the semen concentration, sperm count and progressive motility vitality were markedly decreased in the long-term smoking group. Compared with the non-smoking group, the abnormal head rates in the heavy smoking group and long-term smoking group were significantly increased. The sperm viability and seminal plasma zinc concentration were markedly increased in the smoking group.
\end{abstract}

Correspondence to: Professor Xueqing $\mathrm{Wu}$, Reproductive Medicine Center, Children's Hospital of Shanxi and Women Health Center of Shanxi, 13 Xinmin North Street, Xinghua Ling, Taiyuan, Shanxi 030000, P.R. China

E-mail: 15826161519@163.com

Key words: DNA damage, male infertility, checkpoint kinase 1, semen quality
Increased DNA fragmentation rates were found in the smoking group. The expression of Chk1 was significantly decreased in the smoking group, compared with the non-smoking group. Progressive motility and sperm concentration showed a nonlinear association with the relative mRNA expression of Chk1. However, an inverse association was found between DNA fragmentation rates and the progressive motility and sperm concentration. These data suggested that the decrease of semen quality caused by cigarette smoking was not only correlated with sperm DNA fragmentation rates, but was also correlated with a decline in the expressive level of Chk1. The expression of Chk1 was associated with DNA damage and apoptosis, the reduction of which may lead to decreased sperm repair and increased sperm apoptosis, with a subsequent effect on semen quality.

\section{Introduction}

Infertility is a common disorder affecting some one in seven couples, and subfertility has become a markedly increasing problem in affluent countries, with the most commonly identified cause attributed to 'male factor' (1-3). Several scientific studies have suggested that a decrease in male fertility is frequently associated with smoking, which may cause a decrease in semen quality (4). The inhalation of cigarette smoke leads to absorption of nicotine, carbon monoxide and heavy metals throughout the body, which can end up in the seminal plasma of smokers via various modes of diffusion and active transport $(1,5,6)$. Reports have shown that there is a continuous and substantial number of cell divisions in the sperm cell differentiation and maturation process (7-9). Simultaneously, cigarette smoking affects semen quality, particularly among heavy smokers or those who have smoked for several years (10).

Studies have demonstrated that Chk1 is a Ser/Thr protein kinase, which controls the G2/M phase transition in response to DNA damage (11-13). Following DNA damage, it is released from chromatin and localizes to the cytoplasm, where a portion localizes to interphase centrosomes (14). In turn, activated Chk phosphorylates a number of downstream effectors to trigger pleiotropic cellular responses, including transcription regulation, alteration of energy consumption, cell-cycle arrest or delay, and DNA repair or cell death if the damage is too severe for repair (14). The harmful substances in 
tobacco inhaled by smokers cause DNA damage, which may elevate DNA fragmentation rates $(15,16)$. Increased sperm DNA fragmentation rates have been positively correlated with impaired fertility (17). Thus, the present study aimed to investigate the expression of Chk1 in sperm cells of smoking and non-smoking men, and to further examine the correlation between DNA fragmentation rates and the expression levels of Chk1 with smoking.

\section{Materials and methods}

Study population. The study population consisted of men, who were referred to the Reproductive Medicine Center of Shanxi Women and Infants Hospital (Taiyuan, China) between January 2013 and January 2015. All subjects were of the Han population from Shanxi Province in north China. The study was approved by the ethics committee of the Shanxi Women and Infants Hospital (Taiyuan, China) and the individuals in the relationships provided consent.

The inclusion criteria were as follows: Being the male partner of an infertile couple for a duration of at least 1 year, having regular intercourse, and seeking infertility treatment at the Reproductive Medicine Center, Shanxi Women and Infants Hospital over the study period. A careful history was obtained from each subject to exclude systemic diseases and assess alcohol assumption; careful physical examination was performed, with measurement of testicular size to exclude abnormalities of the external genitalia and cryptorchidism; ultrasonographic examination was performed to exclude varicoceles; microbiological examination and spermioculture were performed to exclude infections; an immunobead binding test was performed to exclude the presence of anti-sperm antibodies; karyotyping was used to exclude any chromosomal abnormality; and genetic examination was performed to exclude $\mathrm{Y}$ chromosome microdeletions and cystic fibrosis gene mutations.

A brief medical history was obtained, primarily by informal interview with the patient, or from the patient's clinical notes or a self-reported questionnaire. According to the standardization of the World Health Organization (WHO) on smoking and associated literature (18-20), 1,218 men were divided into a smoking group (920 cases) and non-smoking group (298 cases). The smoking group was grouped into three groups, according to daily cigarette consumption, as follows: Mild smoking group (256 cases; $\leq 9$ cigarettes/day), moderate smoking group (365 cases; 10-19 cigarettes/day), heavy smoking group ( 299 cases; $\geq 20$ cigarettes/day). The smoking group was grouped into another three groups, according to the number of years of smoking, as follows: Short-term smoker group (268 cases; $\leq 5$ years), medium-term smoker group (282 cases; 5-10 years), long-term smoker group (370 cases; $\geq 10$ years).

Semen collection and analysis. A semen sample was obtained from all subjects via masturbation following 2-7 days of abstinence for routine sperm counts, according to the WHO (2010) criteria (sperm concentration, motility, morphology and viability) (21). Briefly, the ejaculate volumes were estimated by specimen weight, assuming a semen density of $1.0 \mathrm{~g} / \mathrm{ml}$. Sperm concentration, motility and viability were detected using a sperm class analyzer (CASA system; Microoptic S.L., Barcelona, Spain). Sperm motility was also analyzed using the WHO (2010) criteria of progressive motility, non-progressive motility and immotility. In this analysis, the percentage of motile sperm refers to the percentage of sperm with any flagellar movement, whether twitching or progressive. A single technician assessed sperm morphology using the strict methods recommended by the WHO (2010). In addition to the primary measurements of semen quality (sperm concentration, volume and percentage of motile sperm), the CASA system was used to analyze the total sperm count and total motile sperm count.

Sperm viability analysis. Sperm viability was assessed within $30 \mathrm{~min}$ of ejaculation. Analysis was performed using eosin Y staining (Nanjing KeyGen Biotech Co., Ltd., Nanjing, China), for which $1 \mathrm{~g}$ of eosin was dissolved with $1 \mathrm{~g}$ fresh sperm. The percentage of viable sperm, indicated by an unstained sperm head, and non-viable sperm, indicated by staining of the sperm head, was assessed by counting a minimum of 200 spermatozoa. Replicate counts of 200 sperm on each of two slides were performed using a using a CX31 microscope (Olympus Corporation, Tokyo, Japan), which were then repeated if $>5 \%$ difference was found (4).

Ultraviolet spectrophotometric assay for measurement of spermatozoa acrosin activity. The spermatozoa in each group were analyzed for acrosin activity using a Human Spermatozoa Acrosin Activity Quantitative Assay kit (Huakang Biotech, Shenzhen, China), according to manufacturer's protocol. At $24^{\circ} \mathrm{C}$, the quantity of substrate hydrolyzing $1.0 \mu \mathrm{mol}$ BAPNA/min was defined as $1 \mathrm{IU}$ acrosin activity, and determined based on optical density (OD) values: Acrosin activity $\left(\mu \mathrm{IU} / 10^{6}\right.$ spermatozoa $)=\{[($ sample tube OD value - control tube OD value) $\mathrm{x} 2]$ / $(495 \times 7.5)\} \times 10^{6}$. OD values were assessed on a DNM-9602 ultraviolet spectrophotometer (Beijing Perlong New Technology Co., Ltd., Beijing, China).

Colorimetric assay for measurement of seminal plasma zinc. The seminal fluid in each group was analyzed for zinc using a Seminal Plasma Zinc Quantitative Assay kit (Huakang Biotech), according to the manufacturer's protocol. The seminal fluid $(1 \mathrm{ml})$ was centrifuged for $5 \mathrm{~min}$ at $1,000 \mathrm{x} \mathrm{g}$ at $5 \mathrm{~min}$. The supernatant was transferred into a test tube for use in seminal plasma analysis. The sediment was washed with $1 \mathrm{ml}$ physiological saline solution, mixed on an XH-B vortex-type mixer (Jiangsu Kangjian Medical Apparatus Co., Ltd., Taizhou, China) for $30 \mathrm{sec}$, and centrifuged again, as previously. The supernatant was discarded and the sediment was used for zinc determination, rather than using $200 \mu \mathrm{l}$ of liquid sample. The absorbance of the solutions was measured at $490 \mathrm{~nm}$ on aHR801 microplate reader (Shenzhen Highcreation Technology Co., Ltd., Shenzhen, China). The concentration in the sample was determined using the following formula: Seminal plasma zinc $(\mu \mathrm{mol})=$ zinc concentration $(\mathrm{mmol} / \mathrm{l}) \mathrm{x}$ semen volume $(\mathrm{ml})$.

Sperm DNA fragmentation analysis. The analysis of DNA fragmentation was performed in fresh semen using fluorescence staining with a kit supplied by (Huakang Biotech), 
Table I. Comparison of routine semen parameters between non-smokers and smokers grouped according to daily cigarette consumption.

\begin{tabular}{lccccc}
\hline & & & \multicolumn{3}{c}{ Daily cigarette consumption } \\
\cline { 4 - 6 } Parameter & Non-smoking & Smoking & Mild & Moderate & Heavy \\
\hline Cases (n) & 298 & 920 & 256 & 365 & 299 \\
Semen volume (ml) & $3.63 \pm 1.48$ & $3.44 \pm 1.23$ & $3.53 \pm 1.18$ & $3.40 \pm 1.21$ & $3.47 \pm 1.23$ \\
Sperm concentration $\left(\mathrm{x} 10^{6} / \mathrm{ml}\right)$ & $45.38 \pm 24.83$ & $41.57 \pm 21.93$ & $44.41 \pm 21.42$ & $42.62 \pm 25.26$ & $40.13 \pm 24.73$ \\
Sperm count $\left(\mathrm{x} 10^{6}\right)$ & $49.28 \pm 31.29$ & $44.62 \pm 31.94$ & $47.17 \pm 29.96$ & $45.84 \pm 31.24$ & $43.18 \pm 32.61$ \\
Progressive motility $(\%)$ & $27.97 \pm 10.66$ & $18.26 \pm 11.48$ & $26.42 \pm 12.63$ & $19.58 \pm 11.24$ & $15.21 \pm 9.17$
\end{tabular}

Data are presented as the mean \pm standard deviation. Mild, $\leq 9$ cigarettes/day; moderate, $10-19$ cigarettes/day; heavy, $\geq 20$ cigarettes/day.

based on the fluorescence emission from individual sperm, which were stained with acridine orange (AO). AO molecules are intercalated into double-stranded DNA, and green fluorescence is emitted from the sperm nuclei. The DNA in sperm with immature nuclei are readily denatured into single strands and, following AO molecule aggregation in the nuclei, the color of the fluorescence becomes orange-red. The cell suspension was pipetted onto a glass slide and observed under a BX51 fluorescence microscope (Olympus Corporation) with a 480-490 nm filter. The percentages of green (normal DNA integrity) and orange-red (abnormal DNA integrity) spermatozoa in each sample of 200 spermatozoa were calculated by the same examiner. The integrity of sperm nuclear DNA was considered abnormal when the percentage of denaturation (orange-red spermatozoa on AO staining) was $>34 \%$.

Reverse transcription-quantitative polymerase chain reaction $(R T-q P C R)$ analysis. RT-qPCR analysis was used to assess the transcriptional expression of tumor-associated genes, including Chk1. Total RNAs were extracted from the spermatozoa using a Total RNA Purification kit (Nanjing KeyGen Biotech Co., Ltd.), the concentration of the RNA was determined using a NanoDrop 1000 (NanoDrop; Thermo Fisher Scientific, Inc., Wilmington, DE, USA) and reverse transcribed using an Transcriptor First Strand cDNA synthesis kit (Nanjing KeyGen Biotech Co., Ltd.). The reaction was performed in the following conditions: $30^{\circ} \mathrm{C}$ for $10 \mathrm{~min} ; 42^{\circ} \mathrm{C}$ for $30 \mathrm{~min}$; $99^{\circ} \mathrm{C}$ for $5 \mathrm{~min}$; and $5^{\circ} \mathrm{C}$ for $5 \mathrm{~min}$. qPCR was conducted in a CFX-96 (Bio-Rad Laboratories, Inc., Hercules, CA, USA) using a One Step SYBR PrimeScript RT-PCR kit (Takara Bio., Inc.), as described. The specific primers (Invitrogen; Thermo Fisher Scientific, Inc., Waltham, MA, USA) were as follows: Chk1, forward 5'-ATA TGAAGCGTGCCGTAGACT-3' and reverse 5'-TGCCTATGT CTGGCTCTATTCTG-3'; GAPDH, forward 5'-ACCACA GTCCATGCCATCAC-3' and reverse 5'-TCCACCACCCTG TTGCTGTA-3'. qPCR was performed as follows: Initial denaturation at $98^{\circ} \mathrm{C}$ for $5 \mathrm{~min} ; 40$ cycles of $95^{\circ} \mathrm{C}$ for $10 \mathrm{sec}$, and $72^{\circ} \mathrm{C}$ for $15 \mathrm{sec}$. The $2^{-\Delta \Delta \mathrm{Cq}}$ method was used for quantification, calculated according to the manufacturer's protocol, with results expressed as the mean \pm standard deviation.

Western blot analysis. Spermatozoa were prepared from each group for western blot analysis to determine the expression level of the tumor-associated protein, Chk1. The seminal fluid $(1 \mathrm{ml})$ was centrifuged for $5 \mathrm{~min}$ at $1,000 \mathrm{x} \mathrm{g}$. The precipitate was washed with $1 \mathrm{ml}$ physiological saline solution, mixed on a vortex-type mixer for $30 \mathrm{sec}$, and centrifuged again, as above. For analysis of cellular protein levels, spermatozoa cells were rinsed twice with ice-cold phosphate-buffered saline and then lysed in ice-cold lysis buffer [containing $20 \mathrm{mM}$ Tris ( $\mathrm{pH} 7.5$ ), $150 \mathrm{mM} \mathrm{NaCl}, 1 \mathrm{mM}$ ETDA, 1 mM EGTA, 1\% Triton X-100, $2.5 \mathrm{mM}$ sodium pyrophosphate, $1 \mathrm{mM} \beta$-glycerophosphate, $1 \mathrm{mM} \mathrm{Na} 3 \mathrm{VO} 4,1 \mathrm{mM}$ PMSF, and $10 \mu \mathrm{g} / \mathrm{ml}$ each of leupeptin, aprotinin, and pepstatin) for $30 \mathrm{~min}$. Cell lysates were centrifuged at $13,000 \mathrm{x} \mathrm{g}$ for $10 \mathrm{~min}$ at $4^{\circ} \mathrm{C}$ and the protein concentration was determined by the Bradford assay. Proteins $(20 \mu \mathrm{g})$ were loaded onto 8\% SDS-PAGE gels for electrophoresis and then transferred onto nitrocellulose membranes. The transferred nitrocellulose membranes were blocked with 5\% dried skim milk for $1 \mathrm{~h}$ at room temperature, then incubated with mouse anti-Chk1 monoclonal antibody (1:200; Santa Cruz Biotechnology, Inc., Santa Cruz, CA, USA; cat. no. sc-377231) at $4^{\circ} \mathrm{C}$ for $12 \mathrm{~h}$. The membranes were then exposed to goat anti-mouse or rabbit secondary antibody (1:200; Zhongshan Bioengineering, Beijing, China; cat. no. PV9005) in blocking buffer for $1 \mathrm{~h}$ at room temperature. The bands were demonstrated by enhanced chemiluminescence reagents (Santa Cruz Biotechnology, Inc.) for $1 \mathrm{~min}$ and analyzed using Image-Pro Plus 5.1 software (Media Cybernetics, Inc., Rockville, MD, USA).

Statistical analysis. All data were analyzed using SPSS 17.0 (SPSS, Inc. Chicago, IL, USA). Normally distributed data are expressed as the mean \pm standard deviation. $\mathrm{P}<0.05$ was considered to indicate a statistically significant difference. To assess the normality of the distribution, a Shapiro-Wilk test was performed. One-way analysis of variance was used for comparison among multiple groups if the variance was homogeneous, whereas non-normally distributed variables were analyzed using a Mann-Whitney U test or Kruskal-Wallis variance analysis, as appropriate.

\section{Results}

Comparison of semen parameters between smokers and non-smokers. As shown in Tables I and II, routine semen 
Table II. Comparison of routine semen parameters between non-smokers and smokers grouped according to the duration of smoking.

\begin{tabular}{lcccc}
\hline & & \multicolumn{3}{c}{ Duration of smoking } \\
\cline { 3 - 5 } Parameter & Non-smoking & Short-term & Medium-term & Long-term \\
\hline Cases $(\mathrm{n})$ & 298 & 268 & 282 & 370 \\
Semen volume $(\mathrm{ml})$ & $3.63 \pm 1.48$ & $3.57 \pm 1.24$ & $3.43 \pm 1.42$ & $2.12 \pm 1.11$ \\
Sperm concentration $\left(\mathrm{x} 10^{6} / \mathrm{ml}\right)$ & $45.38 \pm 24.83$ & $43.65 \pm 20.39$ & $41.59 \pm 21.11$ & $28.85 \pm 22.22$ \\
Sperm count $\left(\mathrm{x} 10^{6}\right)$ & $49.28 \pm 31.29$ & $48.47 \pm 21.56$ & $44.54 \pm 22.39$ & $37.67 \pm 16.22$ \\
Progressive motility $(\%)$ & $27.97 \pm 10.66$ & $25.67 \pm 11.18$ & $18.69 \pm 12.24$ & $13.28 \pm 11.43$ \\
\hline
\end{tabular}

Data are presented as the mean \pm standard deviation. Short-term, $\leq 5$ years; medium-term, 5-10 years; long-term, $\geq 10$ years.

Table III. Comparison of sperm morphology between non-smokers and smokers grouped according to daily cigarette consumption.

\begin{tabular}{lccccc}
\hline & & \multicolumn{3}{c}{ Daily cigarette consumption } \\
\cline { 5 - 6 } Parameter & Non-smoking & Smoking & Mild & Moderate & Heavy \\
\hline Cases & 298 & 920 & 256 & 365 & 299 \\
Normal sperm & $7.22 \pm 1.49$ & $6.43 \pm 1.33$ & $7.13 \pm 1.38$ & $6.97 \pm 1.55$ & $6.13 \pm 1.07$ \\
Abnormal head & $82.51 \pm 11.66$ & $88.38 \pm 15.11$ & $86.36 \pm 13.10$ & $86.02 \pm 10.16$ & $98.22 \pm 18.54$ \\
Abnormal body & $41.38 \pm 8.58$ & $49.32 \pm 14.43$ & $47.73 \pm 12.89$ & $46.52 \pm 14.81$ & $52.37 \pm 14.23$ \\
Abnormal tail & $6.23 \pm 7.19$ & $11.64 \pm 12.77$ & $9.38 \pm 8.64$ & $10.57 \pm 10.32$ & $12.39 \pm 14.71$ \\
\hline
\end{tabular}

Data are presented as the mean \pm standard deviation. Mild, $\leq 9$ cigarettes/day; moderate, $10-19$ cigarettes/day; heavy, $\geq 20$ cigarettes/day.

parameters in the smoking groups were compared with those of the non-smoking group. No significant differences in semen volume, sperm concentration or sperm count were found between the smoking and non-smoking groups $(\mathrm{P}>0.05)$. However, progressive motility in the smoking group was significantly decreased $(\mathrm{P}<0.05)$, compared with the non-smoking group. No significant changes in routine semen parameters were observed in the mild smoking group $(\mathrm{P}>0.05)$, whereas the moderate and heavy smoking groups had significantly decreased progressive motility $(\mathrm{P}<0.05)$. No significant differences were found in the routine semen parameters in the short-term smoking group, compared with the non-smoking group $(\mathrm{P}>0.05)$, whereas the medium-term smoking group had significantly decreased progressive motility $(\mathrm{P}<0.05)$, and the long-term smoking group had decreased semen concentration, sperm count and progressive motility $(\mathrm{P}<0.05)$.

Comparison of sperm morphology between smokers and non-smokers. As shown in Tables III and IV, the sperm morphology in the smoking groups was compared with the non-smoking group. No significant differences were found in the sperm morphology in the normal sperm count and abnormal head, body and tail counts between the smoking and non-smoking groups $(\mathrm{P}>0.05)$. Compared with the non-smoking group, no significant changes were found in the sperm morphology between the mild smoking group and moderate smoking group $(\mathrm{P}>0.05)$, nor were there significant differences between the short-term smoking group and medium-term smoking group $(\mathrm{P}>0.05)$. However, the abnormal head rate in the heavy smoking group and long-term smoking group were significantly higher, compared with those in the non-smoking group $(\mathrm{P}<0.05)$.

Comparison of sperm viability between smokers and non-smokers. Sperm viability was analyzed using eosin $\mathrm{Y}$ staining. As shown in Fig. 1A, the viable sperm, in which sperm heads remained unstained, and non-viable sperm, in which sperm heads were stained, were examined using a CX31 microscope (Olympus Corporation). Compared with the non-smoking group, the smoking group had significantly decreased sperm viability (Fig. 1B; $\mathrm{P}<0.05)$.

Comparison of plasma zinc, spermatozoa acrosin activity and DNA fragmentation rates between smokers and non-smokers. As shown in Fig. 2A, the seminal plasma zinc concentration decreased significantly in the smoking group, compared with the non-smoking group $(\mathrm{P}<0.05)$. Similar results were obtained for spermatozoa acrosin activity between the smoking group and non-smoking group (Fig. 2B; $\mathrm{P}<0.05$ ). DNA fragmentation rates were analyzed using $\mathrm{AO}$ staining. As shown in Fig. 2C, the green (normal DNA integrity) and orange-red (abnormal DNA integrity) spermatozoa were examined using a BX51 fluorescence microscope (Olympus Corporation) with a 480-490 nm filter. 
Table IV. Comparison of sperm morphology between non-smokers and smokers grouped according to duration of smoking.

\begin{tabular}{lcccc}
\hline & & \multicolumn{3}{c}{ Duration of smoking } \\
\cline { 3 - 5 } Parameter & Non-smoking & Short-term & Medium-term & 370 \\
\hline Cases (n) & 298 & 268 & 282 & $5.29 \pm 1.27$ \\
Normal sperm & $7.22 \pm 1.38$ & $7.42 \pm 1.23$ & $6.29 \pm 1.26$ & $99.43 \pm 16.28$ \\
Abnormal head & $82.51 \pm 10.62$ & $80.91 \pm 14.25$ & $88.27 \pm 12.77$ & $52.50 \pm 9.71$ \\
Abnormal body & $41.38 \pm 9.48$ & $47.88 \pm 8.76$ & $51.93 \pm 10.36$ & $11.31 \pm 12.19$ \\
Abnormal tail & $6.23 \pm 7.44$ & $11.59 \pm 8.61$ & $9.37 \pm 11.27$ &
\end{tabular}

Data are presented as the mean \pm standard deviation. Short-term, $\leq 5$ years; medium-term, 5-10 years; long-term, $\geq 10$ years.

Table V. Association between DNA fragmentation rates and the expression of Chk1 with semen parameters in all participants.

\begin{tabular}{|c|c|c|c|c|c|}
\hline \multirow[b]{2}{*}{ Parameter } & \multirow[b]{2}{*}{ Mean \pm SD } & \multicolumn{2}{|c|}{$\begin{array}{c}\text { Relative expression } \\
\text { of Chk1 }\end{array}$} & \multicolumn{2}{|c|}{$\begin{array}{l}\text { DNA fragmentation } \\
\text { rate }\end{array}$} \\
\hline & & r-value & P-value & r-value & P-value \\
\hline Relative expression of Chk1 & $1.43 \pm 0.28$ & - & - & - & - \\
\hline DNA fragmentation rate & $0.58 \pm 0.01$ & - & - & - & - \\
\hline Progressive motility (\%) & $22.16 \pm 9.32$ & 0.042 & $0.027^{\mathrm{a}}$ & 0.042 & $0.027^{\mathrm{a}}$ \\
\hline Sperm concentration $\left(\mathrm{x} 10^{6} / \mathrm{ml}\right)$ & $39.27 \pm 15.22$ & 0.047 & $0.026^{\mathrm{a}}$ & 0.037 & $0.012^{\mathrm{a}}$ \\
\hline
\end{tabular}

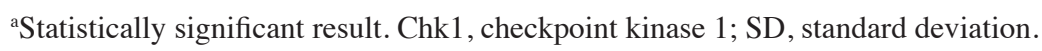
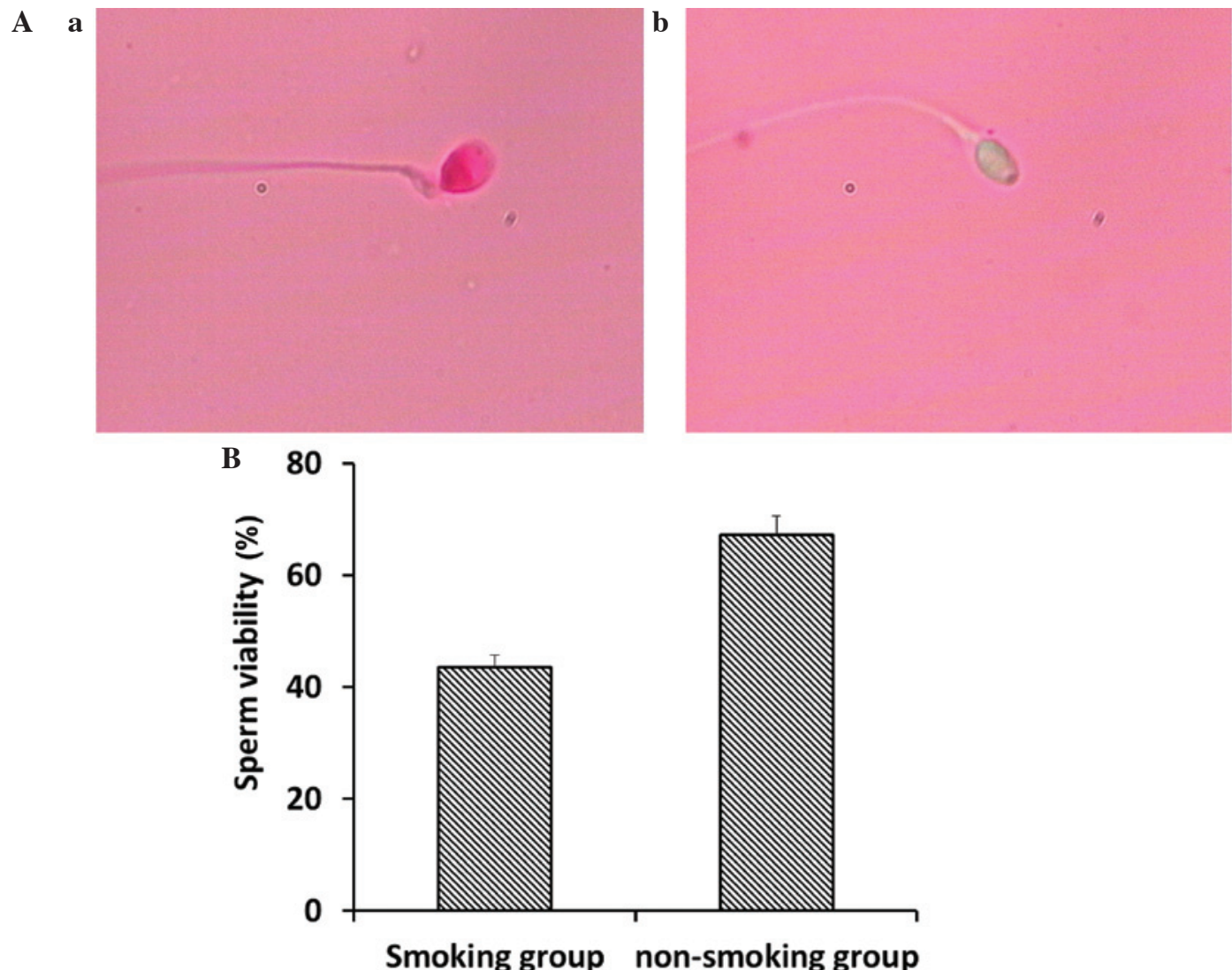

Figure 1. Comparison of sperm viability between smokers and non-smokers. (A) Sperm viability was analyzed using eosin Y staining. The (a) non-viable sperm, indicated by a stained head, and (b) viable sperm indicated by an unstained head, were examined using microscopy (magnification, x20). (B) Sperm viability in the smoking group was significantly decreased, compared with that in the non-smoking group. $(\mathrm{P}<0.05)$. Data are presented as the mean \pm standard deviation. 


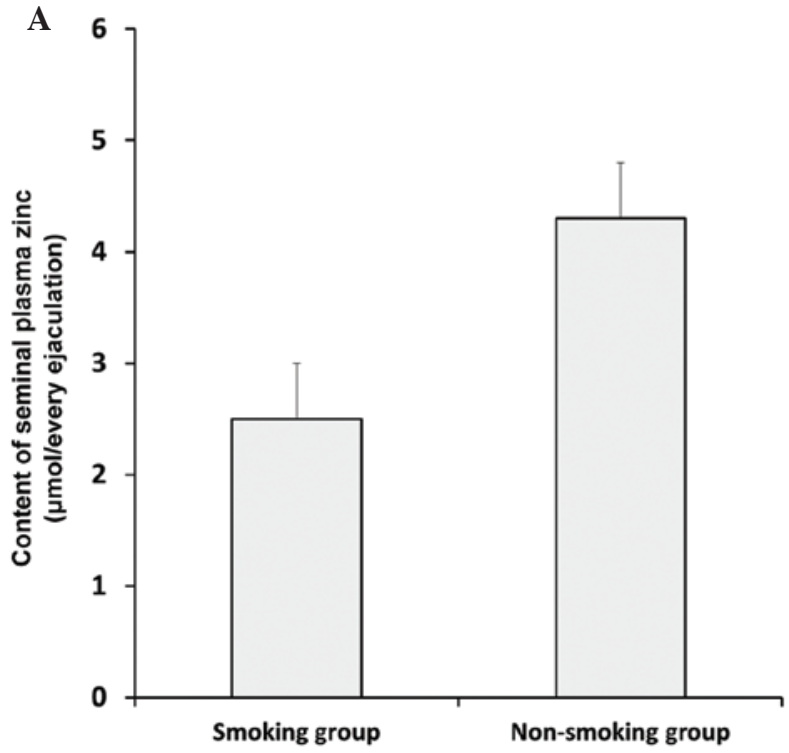

B
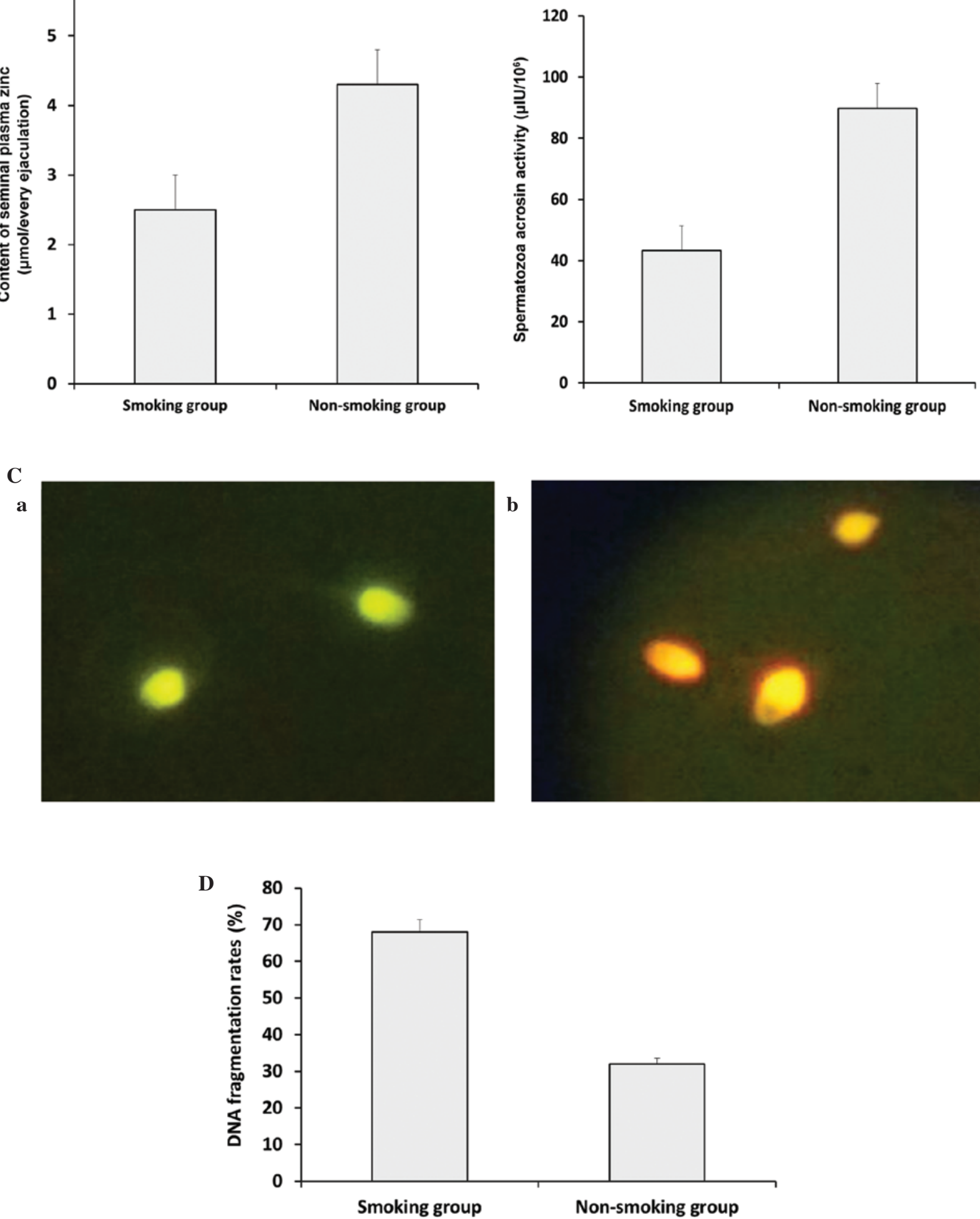

Figure 2. Comparison of plasma zinc concentration, spermatozoa acrosin activity and DNA fragmentation rates between the non-smoking and smoking groups (A) Seminal plasma zinc concentration decreased significantly in the smoking group, compared with the non-smoking group $(\mathrm{P}<0.05)$. (B) Spermatozoa acrosin activity decreased significantly in the smoking group, compared with the non-smoking group $(\mathrm{P}<0.05)$. (C) DNA fragmentation rates were analyzed using acridine orange staining. The (a) green (normal DNA integrity) and (b) orange-red (abnormal DNA integrity) spermatozoa were examined using a BX51 Olympus fluorescence microscope with a 480-490 nm filter (magnification, x20). (D) A significant increase in DNA fragmentation rate was observed in the smoking group, compared with the non-smoking group $(\mathrm{P}<0.05)$. Data are presented as the mean \pm standard deviation.

Compared with the non-smoking group, the smoking group exhibited a significantly higher DNA fragmentation rate (Fig. 2D; $\mathrm{P}<0.05$ ).
Expression levels of Chkl in the sperm of smokers and non-smokers. Analyses using RT-qPCR (Fig. 3A) and western blot analyses (Fig. 3B) demonstrated that the expression of 
A

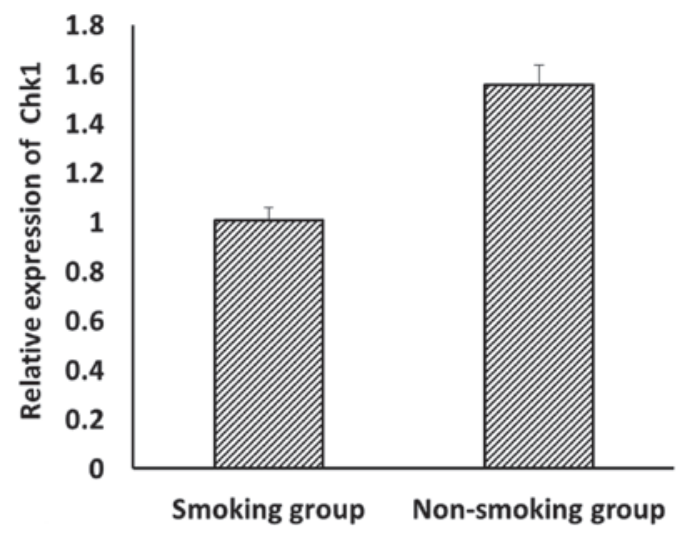

B

\section{Smoking group Non-smoking group}

Chk1

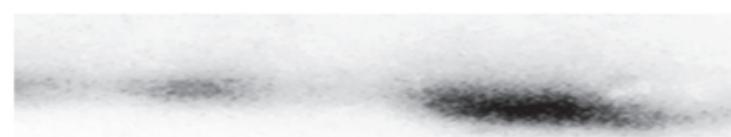

GAPDH

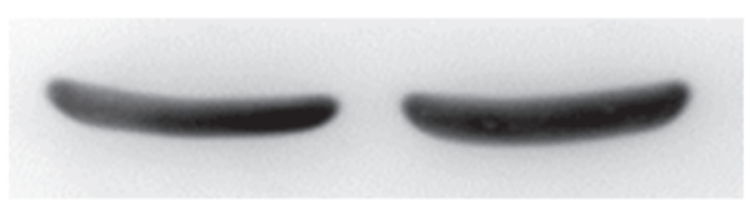

C

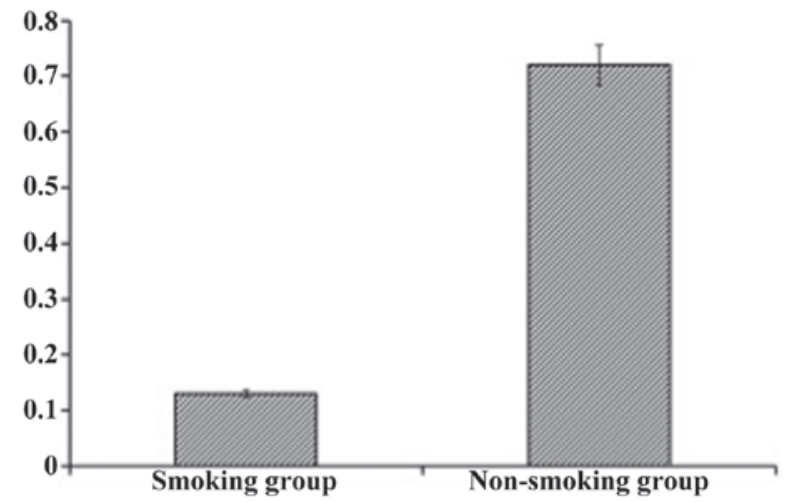

Figure 3. Expression levels of Chk1 in the sperm of smokers and non-smokers. (A) Expression levels of Chk1 were analyzed using reverse transcription-quantitative polymerase chain reaction analysis, which demonstrated that the mRNA expression of Chk1 was significantly decreased in the smoking group, compared with the non-smoking group $(\mathrm{P}<0.05)$. Data are presented as the mean \pm standard deviation. (B) Western blot analysis and $(\mathrm{C})$ quantification showed that the protein expression levels of Chk1 were significantly decreased in the smoking group, compared with the non-smoking group $(\mathrm{P}<0.05)$. Chk1, checkpoint kinase 1.

Chk1 was significantly decreased in the smoking group, compared with the non-smoking group $(\mathrm{P}<0.05)$.

Association between DNA fragmentation rates and Chk1 with semen parameters. The present study found a non-linear association between the relative mRNA expression of Chk1, and the progressive motility and sperm concentration $(\mathrm{P}<0.05)$. However, DNA fragmentation rates were inversely association $(\mathrm{P}<0.05)$ with progressive motility and sperm concentration (Table V).

\section{Discussion}

Cigarette smoking is a recognized health hazard, and the highest prevalence of smokers is in young men of reproductive age (22). There is considerable evidence that cigarette smoking has a major role in the etiology of male infertility (23). Smokers inhale several toxins, including nicotine, carbon monoxide and other mutagenic compounds (24). Cigarette smoking has been associated with detrimental effects on sperm morphology, density and motility (5). The mechanisms through which smoking by men may be linked to detrimental effects on reproduction and sperm parameters remain to be fully elucidated. Direct biological and toxic effects are possible on male sperm cells a. The inhalation of cigarette smoke leads to the absorption of nicotine, carbon monoxide, cadmium and other mutagenic compounds, which may reach the male reproductive system and cause alterations, including altered antioxidant concentrations, reactive oxygen species generation, aneuploidy rates, and DNA damage in spermatozoa and semen $(5,10,22)$. In addition, it has been found that heavy smoking-induced DNA damaged is associated with abnormal spermatozoa and male infertility $(25,26)$. Thus, it is necessary to evaluate the effects of cigarette smoking on DNA damage and repair mechanisms in sperm.

In the present study, it was observed that the progressive motility of the sperm in the moderate and heavy smoking groups were significantly decreased, compared with the non-smokers, whereas no significant changes were observed in the mild smoking group. No significant differences were observed in the routine semen parameters of the short-term smoking group, compared with the non-smoking group. However, the sperm in the medium-term smoking group had significantly decreased progressive motility, and the long-term smoking group had decreased semen concentration, sperm count and progressive motility, compared with the non-smoking group. Compared with the non-smoking group, no significant changes were found in the sperm morphology in the mild smoking group or moderate smoking group. This was also the case for the short-term smoking group and medium-term smoking group. However, the abnormal head rates in the heavy smoking group and long-term 
smoking group showed significant increases, compared with the non-smoking group. Compared with the non-smoking group, the smoking group exhibited a significant increase in sperm viability. The seminal plasma zinc concentration decreased significantly in the smoking group, compared with non-smoking group, and the smoking group had significantly increased DNA fragmentation rates, compared with the non-smoking group. Similar results were obtained for spermatozoa acrosin activity between the smoking group and non-smoking group. These data are consistent with the results of previous studies $(25,26)$.

It has been reported that, in response to DNA damage, the activation of the Chk1 facilitates S and G2 checkpoint arrest (27-29), and it may promote the survival of cells in the presence of DNA damage-inducing agents. Activated Chk1 phosphorylates a number of downstream effectors to trigger a pleiotropic cellular response, which includes transcription regulation, alterations in energy consumption, cell-cycle arrest or delay and DNA repair or cell death if the damage is too severe for repair (30-32).

In the present study, the expression of Chk1 was significantly decreased in the smoking group, compared with the non-smoking group. There was a nonlinear association between the relative mRNA expression of Chk1 and the progressive motility and sperm concentration. However, an inverse association was found between DNA fragmentation rates and the progressive motility and sperm concentration. These data suggested that the decrease of semen quality caused by cigarette smoking was not only correlated with sperm DNA fragmentation indices, but was also correlated with a decline in the expression of Chk1. The expression of Chk1 was correlated with sperm DNA damage and apoptosis, and its reduction may lead to decreased sperm repair and increased sperm apoptosis, with a subsequent effect on semen quality.

In conclusion, the results obtained in the present study provide useful information regarding the expression of Chk1 in sperm cells of smoking and non-smoking men, and the association between DNA fragmentation rates and the expression levels of Chk1 with smoking. They may also offer information for the prevention and treatment of male infertility as a result of smoking.

\section{Acknowledgements}

The present study was supported by the Research Fund of National Health and Family Planning Commission of China (grant no. RFNHFPCC, 201402004).

\section{References}

1. Jo J, Lee SH, Lee JM and Jerng UM: Semen quality improvement in a man with idiopathic infertility treated with traditional Korean medicine: A case report. Explore (NY) 11: 320-323, 2015.

2. Agarwal A, Mulgund A, Hamada A and Chyatte MR: A unique view on male infertility around the globe. Reprod Biol Endocrinol 13: 37, 2015.

3. Gilany K, Minai-Tehrani A, Savadi-Shiraz E, Rezadoost H and Lakpour N: Exploring the human seminal plasma proteome: An unexplored gold mine of biomarker for male infertility and male reproduction disorder. J Reprod Infertil 16: 61-71, 2015.

4. Samplaski MK, Dimitromanolakis A, Lo KC, Grober ED, Mullen B, Garbens A and Jarvi KA: The relationship between sperm viability and DNA fragmentation rates. Reprod Biol Endocrinol 13: 42, 2015.
5. Abdul-Ghani R, Qazzaz M, Dabdoub N, Muhammad R and Abdul-Ghani AS: Studies on cigarette smoke induced oxidative DNA damage and reduced spermatogenesis in rats. J Environ Biol 35: 943-947, 2014.

6. Hamad MF, Shelko N, Kartarius S, Montenarh M and Hammadeh ME: Impact of cigarette smoking on histone (H2B) to protamine ratio in human spermatozoa and its relation to sperm parameters. Andrology 2: 666-677, 2014.

7. Ahmadnia H, Ghanbari M, Moradi MR and Khaje-Dalouee M: Effect of cigarette smoke on spermatogenesis in rats. Urol $\mathrm{J} 4$ : 159-163, 2007.

8. Shukla KK, Mahdi AA and Rajender S: Apoptosis, spermatogenesis and male infertility. Front Biosci (Elite Ed) 4: 746-754, 2012.

9. Sun QY, Breitbart H and Schatten H: Role of the MAPK cascade in mammalian germ cells. Reprod Fertil Dev 11: 443-450, 1999.

10. Tian M, Bao H, Martin FL, Zhang J, Liu L, Huang Q and Shen H: Association of DNA methylation and mitochondrial DNA copy number with human semen quality. Biol Reprod 91: $101,2014$.

11. Albiges L, Goubar A, Scott V, Vicier C, Lefèbvre C, Alsafadi S, Commo F, Saghatchian M, Lazar V, Dessen P, et al: Chk1 as a new therapeutic target in triple-negative breast cancer. Breast 23: 250-258, 2014.

12. Al-Kaabi MM, Alshareeda AT, Jerjees DA, Muftah AA, Green AR, Alsubhi NH, Nolan CC, Chan S, Cornford E, Madhusudan S, et al: Checkpoint kinase1 (CHK1) is an important biomarker in breast cancer having a role in chemotherapy response. Br J Cancer 112: 901-911, 2015.

13. Ma YC, Su N, Shi XJ, Zhao W, Ke Y, Zi X, Zhao NM, Qin YH, Zhao HW and Liu HM: Jaridonin-induced G2/M phase arrest in human esophageal cancer cells is caused by reactive oxygen species-dependent Cdc2-tyr15 phosphorylation via ATM-Chk1/2-Cdc25C pathway. Toxicol Appl Pharmacol 282: 227-236, 2015.

14. Zuazua-Villar P, Rodriguez R, Gagou ME, Eyers PA and Meuth M: DNA replication stress in CHK1-depleted tumour cells triggers premature (S-phase) mitosis through inappropriate activation of Aurora kinase B. Cell Death Dis 5: e1253, 2014.

15. Sepaniak S, Forges T, Fontaine B, Gerard H, Foliguet B, Guillet-May F, Zaccabri A and Monnier-Barbarino P: Negative impact of cigarette smoking on male fertility: From spermatozoa to the offspring. J Gynecol Obstet Biol Reprod (Paris) 33: 384-390, 2004 (In French).

16. Silver EW, Eskenazi B, Evenson DP, Block G, Young S and Wyrobek AJ: Effect of antioxidant intake on sperm chromatin stability in healthy nonsmoking men. J Androl 26: 550-556, 2005.

17. Ozkosem B, Feinstein SI, Fisher AB and O'Flaherty C: Advancing age increases sperm chromatin damage and impairs fertility in peroxiredoxin 6 null mice. Redox Biol 5: 15-23, 2015.

18. Pasqualotto FF, Sobreiro BP, Hallak J, Pasqualotto EB and Lucon AM: Cigarette smoking is related to a decrease in semen volume in a population of fertile men. BJU Int 97: 324-326, 2006.

19. Zhou YL, Chen K, Yu BL and Gao XC: Cigarette smoking decreases sperm nucleoprotein transition in infertile males. Zhonghua Nan Ke Xue 19: 794-797, 2013 (In Chinese).

20. Trummer H, Habermann H, Haas J and Pummer K: The impact of cigarette smoking on human semen parameters and hormones. Hum Reprod 17: 1554-1559, 2002.

21. Lu JC, Huang YF and Lü NQ: WHO laboratory manual for the examination and processing of human serum: Its applicability to andrology laboratories in China. Zhonghua Nan Ke Xue 16: 867-871, 2010.

22. Sobinoff AP, Sutherland JM, Beckett EL, Stanger SJ, Johnson R, Jarnicki AG, McCluskey A, St John JC, Hansbro PM and McLaughlin EA: Damaging legacy: Maternal cigarette smoking has long-term consequences for male offspring fertility. Hum Reprod 29: 2719-2735, 2014.

23. Davar R, Sekhavat L and Naserzadeh N: Semen parameters of non-infertile smoker and non-smoker men. J Med Life 5: 465-468, 2012.

24. Fariello RM, Pariz JR, Spaine DM, Gozzo FC, Pilau EJ, Fraietta R, Bertolla RP, Andreoni C and Cedenho AP: Effect of smoking on the functional aspects of sperm and seminal plasma protein profiles in patients with varicocele. Hum Reprod 27: 3140-3149, 2012 
25. Dai JB, Wang ZX and Qiao ZD: The hazardous effects of tobacco smoking on male fertility. Asian J Androl 17: 954-960, 2015.

26. La Maestra S, De Flora S and Micale RT: Effect of cigarette smoke on DNA damage, oxidative stress, and morphological alterations in mouse testis and spermatozoa. Int J Hyg Environ Health 218: 117-122, 2015.

27. Bryant C, Rawlinson R and Massey AJ: Chk1 inhibition as a novel therapeutic strategy for treating triple-negative breast and ovarian cancers. BMC Cancer 14: 570, 2014.

28. Bryant C, Scriven K and Massey AJ: Inhibition of the checkpoint kinase Chk1 induces DNA damage and cell death in human Leukemia and Lymphoma cells. Mol Cancer 13: 147, 2014.
29. Han X, Aslanian A, Fu K, Tsuji T and Zhang Y: The interaction between checkpoint kinase 1 (Chk1) and the minichromosome maintenance (MCM) complex is required for DNA damage-induced Chk1 phosphorylation. J Biol Chem 289: 24716-24723, 2014.

30. González Besteiro MA and Gottifredi V: The fork and the kinase: A DNA replication tale from a CHK1 perspective. Mutat Res Rev Mutat Res 763: 168-180, 2015.

31. Goto H, Kasahara K and Inagaki M: Novel insights into Chk1 regulation by phosphorylation. Cell Struct Funct 40: 43-50, 2015.

32. Grabocka E, Commisso C and Bar-Sagi D: Molecular pathways: Targeting the dependence of mutant RAS cancers on the DNA damage response. Clin Cancer Res 21: 1243-1247, 2015. 